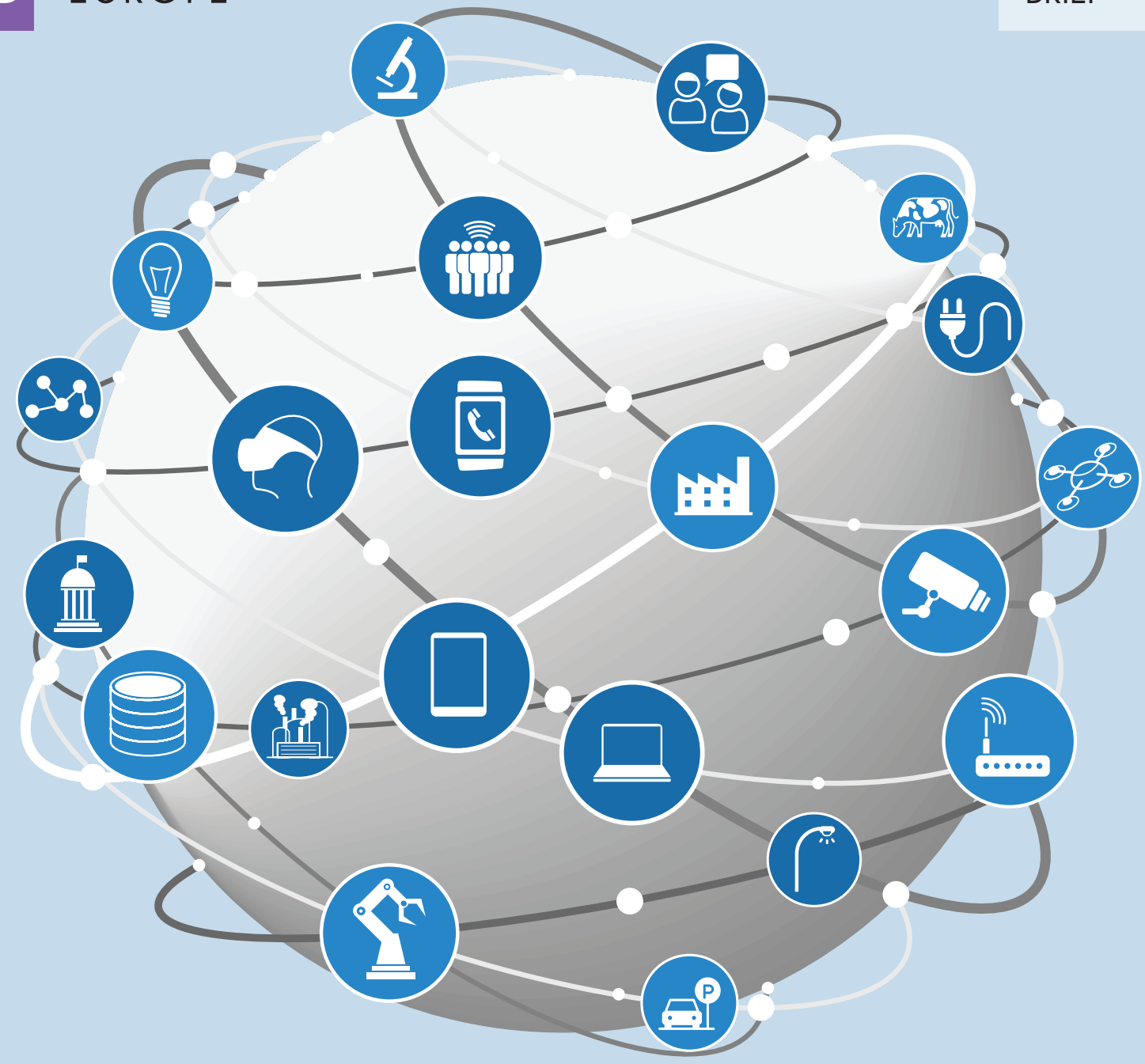

\title{
Accelerating the Internet of Things in the UK Linking policy with practice
}

There is enormous growth in the number of 'things' that incorporate sensors to capture data, photos and video and that can interact with people, IT systems and other things directly using Internet connectivity. Devices with these capabilities are becoming commonplace in homes, streets, cars and workplaces. The potential benefits and risks that arise have resulted in the Internet of Things (IOT) emerging as a critical area of interest to policymakers. Many countries, including the UK, now regard the IOT as highly significant for achieving economic growth. Additionally, consumers have a growing awareness of the connected devices and sensors that are a part of the loT, mainly through their domestic equipment, such as smart TVs and Internet-accessible home security systems and control systems for heating and lighting. It is evident that the loT holds the potential for major economic opportunities across a wide variety of consumer and industrial sectors; however, there are important policy issues that affect the development and adoption of the loT across these sectors. 


\section{The research}

With the loT continuing to grow and develop at a rapid pace, there is a need to use evidence from real world examples of loT implementations and to understand consumer attitudes in order to help inform policies around the IoT. The central aim of our study, commissioned by loTUK and BCS, The Chartered Institute for IT, was to support a process for policy feedback that will inform the development and adoption of the loT in the UK. In order to achieve this central aim and to gain a rounded picture of the potential policy implications of loT developments in the UK, we adopted a bottom-up approach that allowed us to develop a better idea of what is happening 'on the ground' in the UK through insights from businesses and informed users of technology.<smiles>Cc1ccccc1</smiles>

\section{Real world IoT} case studies

We looked at nine real world examples of IoT implementations in the UK that were previously identified by loTUK, and we examined their policy implications. These case studies involved consumer and industrial applications across a wide range of sectors, such as healthcare, energy and environment, transport, retail and agriculture, and they represent examples of applications that have moved from development to deployment. Studying specific loT case studies in depth offers a way to understand what is happening at the frontier of loT industrial activity in the UK.

\section{$\left[\begin{array}{l}-x \\ Z_{x} \\ -x\end{array}\right.$ \\ Survey of informed users of technology}

We carried out a focussed online survey of informed users of technology to gauge their awareness and understanding of the key policy issues related to the advancement of the loT in the UK. The survey helped us gain a sense of what a sector of the public that is familiar with the technology and its implications thinks about such issues as the applicability of IoT to specific sectors, the benefits and risks of using IOT-related products and services, and the role of government in supporting the loT as a strategic industry in the UK.

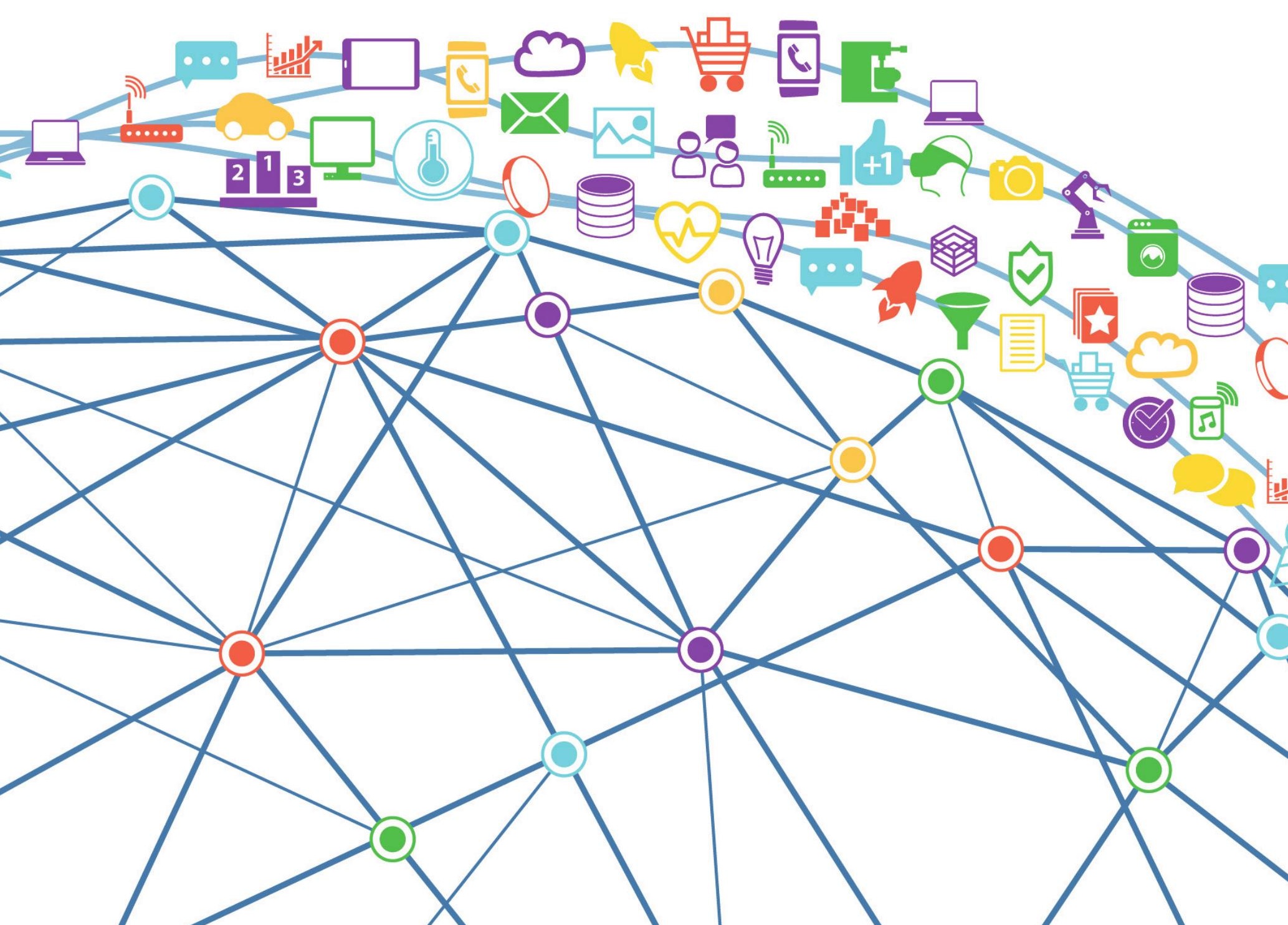




\section{Geographic locations of the nine loT-related case studies examined in the study}

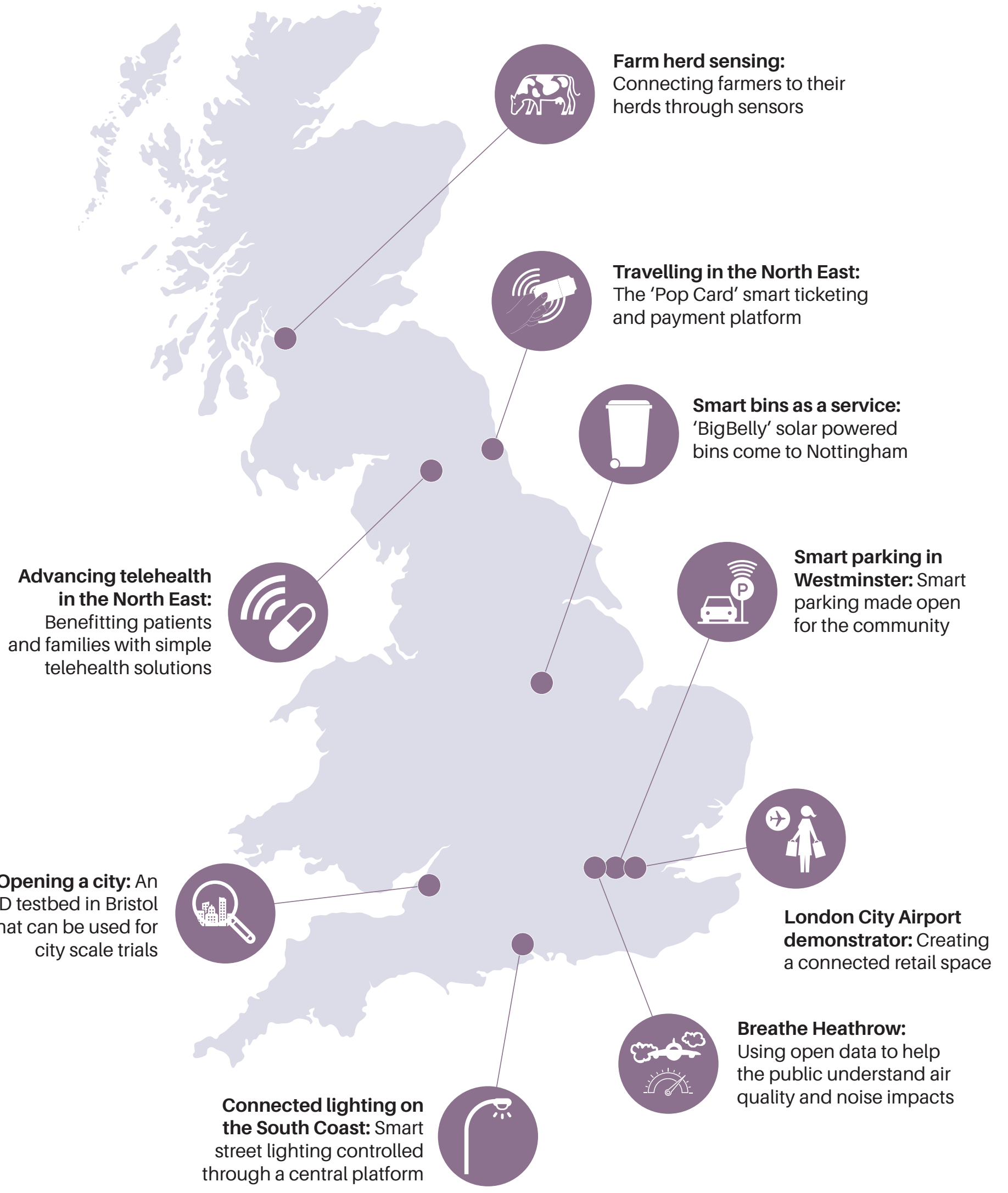


The findings from our research present a number of opportunities and challenges for the development and adoption of the loT in the UK.

\section{Opportunities from case studies}<smiles>O=COCCOCCO</smiles>
Clear, unambiguous and standardised processes for personal data governance are considered to be prerequisites for linking up systems and for making them interoperable and trustworthy.

ini Non-technical factors, such as collaborative networks, organisational capabilities and culture, and citizen engagement, were identified as being important enabling factors for businesses.

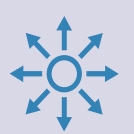

The public sector as a strategic purchaser of new technologies could drive the uptake of the loT. However, it would need to ensure that the small and medium enterprises (SME) leading loT markets can participate and are assessed appropriately in procurement processes.

\section{Challenges from case studies}

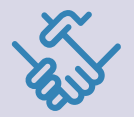

Creating both trust and confidence in the security of the data and processes enabled by the loT is not always aligned with businesses' objectives to innovate quickly and deliver value.

Market uptake and business modelrelated factors are highlighted as key challenges to the growth of the loT market. In particular, the need to demonstrate a sustainable business model with a solid return on investment poses a significant barrier to businesses.

There are mixed perceptions among loT innovators on the ability and level of impact of public policy to drive and accelerate the loT market.

\section{Opportunities from survey}

There is a perception that the public sector could play a stronger role in accelerating the uptake of the loT in the UK, but that it should put citizens at the forefront of these efforts. The priorities for support are seen to be in ensuring interoperability, investing in people (e.g. through skills, training or education), and fostering multistakeholder collaborations (e.g. among businesses, universities and government).

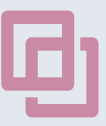

\section{More transparency among} organisations collecting and using data, as well as increased user control and digital literacy, are perceived as key priorities to enable trust and confidence in data sharing and governance.

\section{From the user perspective,} increased environmental sustainability and improved efficiencies for organisations are seen to be the most significant benefits of the loT.

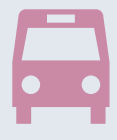

The sectors viewed as most likely to benefit from the loT are transport and logistics, energy and environment, home, and healthcare.

\section{Challenges from survey}

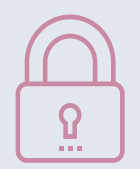

Privacy concerns and security vulnerabilities from increased connectivity, especially the misuse of personal data and the potential to compromise the integrity of business networks, are seen to be key barriers to the wider adoption of the loT. 


\section{Topics for policy discussion and key questions}

Based on these opportunities and challenges, our study attempted to support policy communities (including national and local government policymakers, industry, innovators, academia and the public) through describing a set of wide-ranging policy objectives and associated priority topics for further discussion and exploration. Specifically, we identified four themes for action, aimed at:
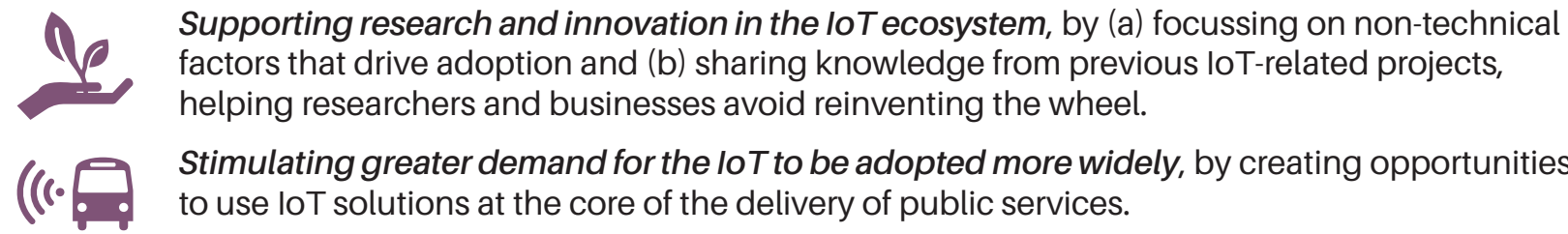

Stimulating greater demand for the loT to be adopted more widely, by creating opportunities to use loT solutions at the core of the delivery of public services.

Strengthening infrastructure and framework conditions for the development and adoption of the loT as a systemic innovation, by (a) promoting greater interoperability and information sharing across applications and (b) supporting the use of integrated loT infrastructure across sectoral boundaries to help scalability.

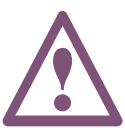

Mitigating the risks of a pervasive loT, by (a) supporting a trusted, people-centric loT ecosystem and (b) addressing concerns about the potential risks of loT technologies to critical national infrastructure.

The infographic shown overleaf depicts each of the proposed priority topics for consideration, clustered by policy objective, along with some supporting policy questions. These questions are wide ranging, and they horizontally apply across different sectors and industries. The required responses to these questions are unlikely to be achieved by public policy or industry alone; instead, they may require an active, multistakeholder approach.

Development of the loT should involve people who are actually affected by the technology.

Our product started with a problem and then we looked at how technology can address this problem.

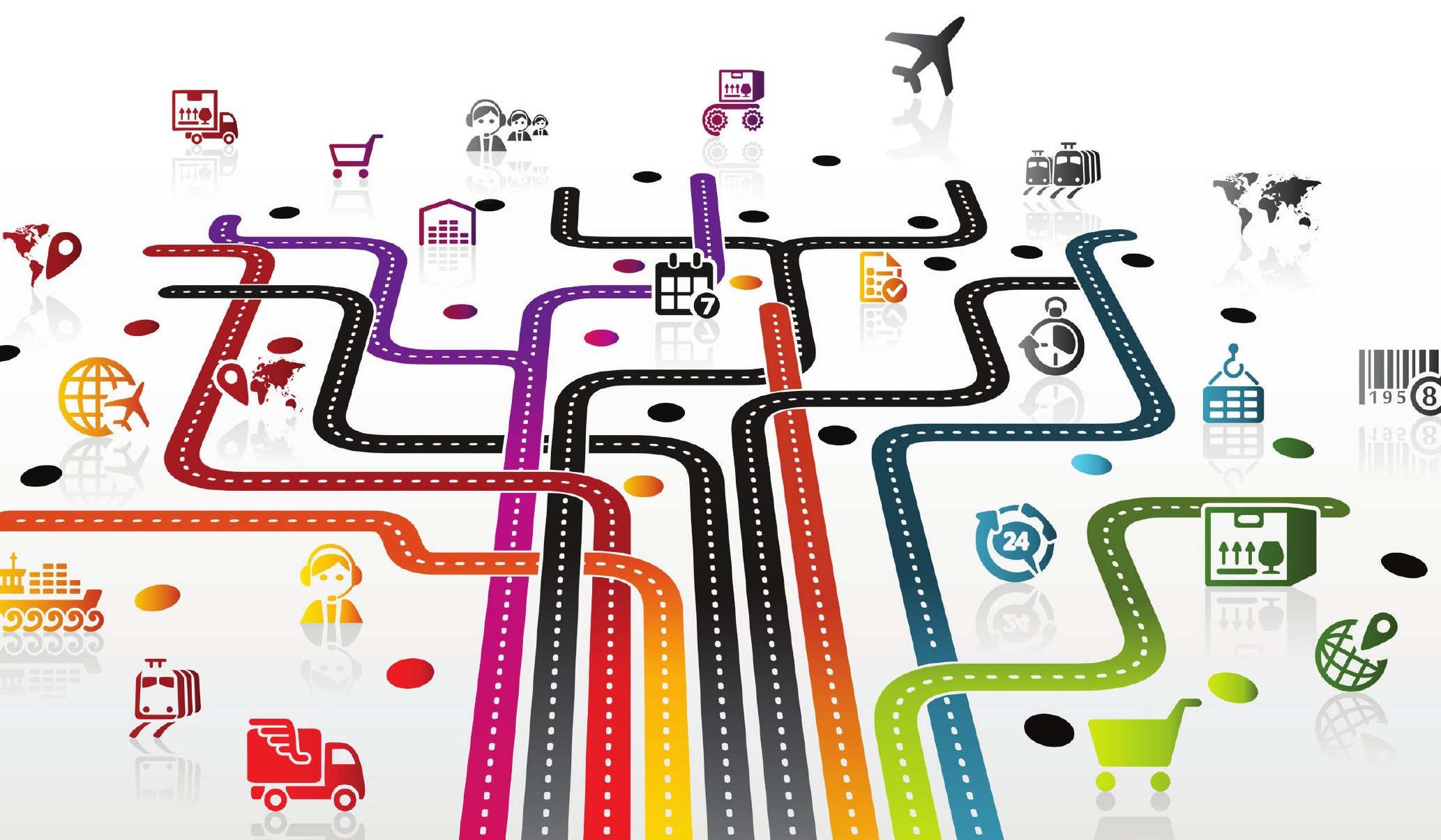


How can policy provide or incentivise more investment in non-technical
innovations? work together to ensure that tested technolitives applied to new contexts and that system-wide effects are realised?

What can the policy community do to support the systematic assessment of risks associated with innovart
their deployment in public infrastructure?

How can current contingency plans be enhanced to identify and manage security risks associated with a growing and pervasive loT?

How can the policy community help
industry balance economic objectives
with creating an loT ecosystem that is
more open, trustworthy and inclusive?
How can the recognised processes
for certifying devices be adapted to
deal with the specific trust challenges
posed by the loT, including consent
and information governance?
How can the policy community
incentivise industry to adopt people-
centric design and development?
What steps can be taken to raise
cyber awareness and educate citizens
about the potential benefits and risks
associated with the loT?

Is there a need to raise awareness among public authorities as to the wider, system
level benefits that could potentially be accrued by leveraging loT technologies as systemic innovations in public infrastructure projects?

How can the policy community support integrated, interoperable loT infrastructure solutions rather than the continued deployment of individual technologies?

How can public authorities frame their requirements to encourage loT standardscompliant devices and services?

How can the policy community support for procurement against public sector funding?
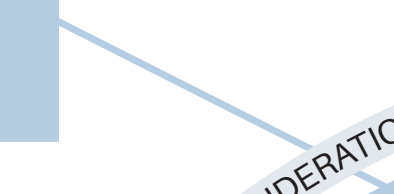

What steps can be taken by the policy community to create opportunities for effective collaborative networks How can the policy community he How can the policy com munty help to develop and the appropriate technical and commercial skills?

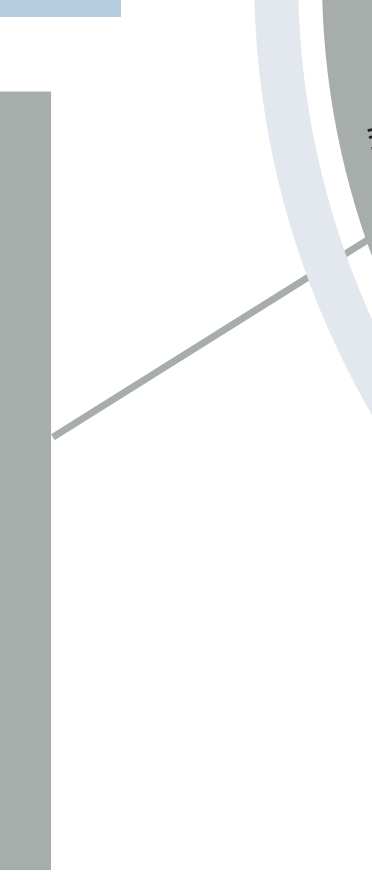

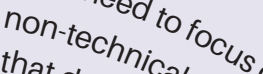

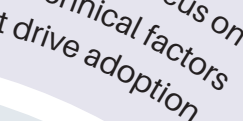

How can the public sector and industry systematically recognise loT-related projects from implemented projects, starting with those that have been funded by government? What are the ways to manner to the various stakeholders in the emerging IOT marketplace? systematically map the loT anticipate and identify areas for public and private research and innovation investment more strategically? for industry to share the lessons of loT implementations?
How can the policy community

What incentives can be created project and system levels?

(1)

What can the policy community do to help accelerate the development of interoperable standards in loT nationally and internationally?

How can publicly funded smart city and large-scale demonstrator projects support the drive towards common standards?

How can public procurement processes support the use of open loT-enabling

How can the policy community capture evidence of the effectiveness and impact of local authorities' procuring of new loT technologies at the

What are the challenges faced by procurement authorities in purchasing LT Technologies with limited evidence of benefits, and how can these

How can public authorities ensure that the procurement processes for IOT technologies balance recognition of innovative, new-to-market SME

How can the policy community support the use of loT technologies for infrastructure projects? 


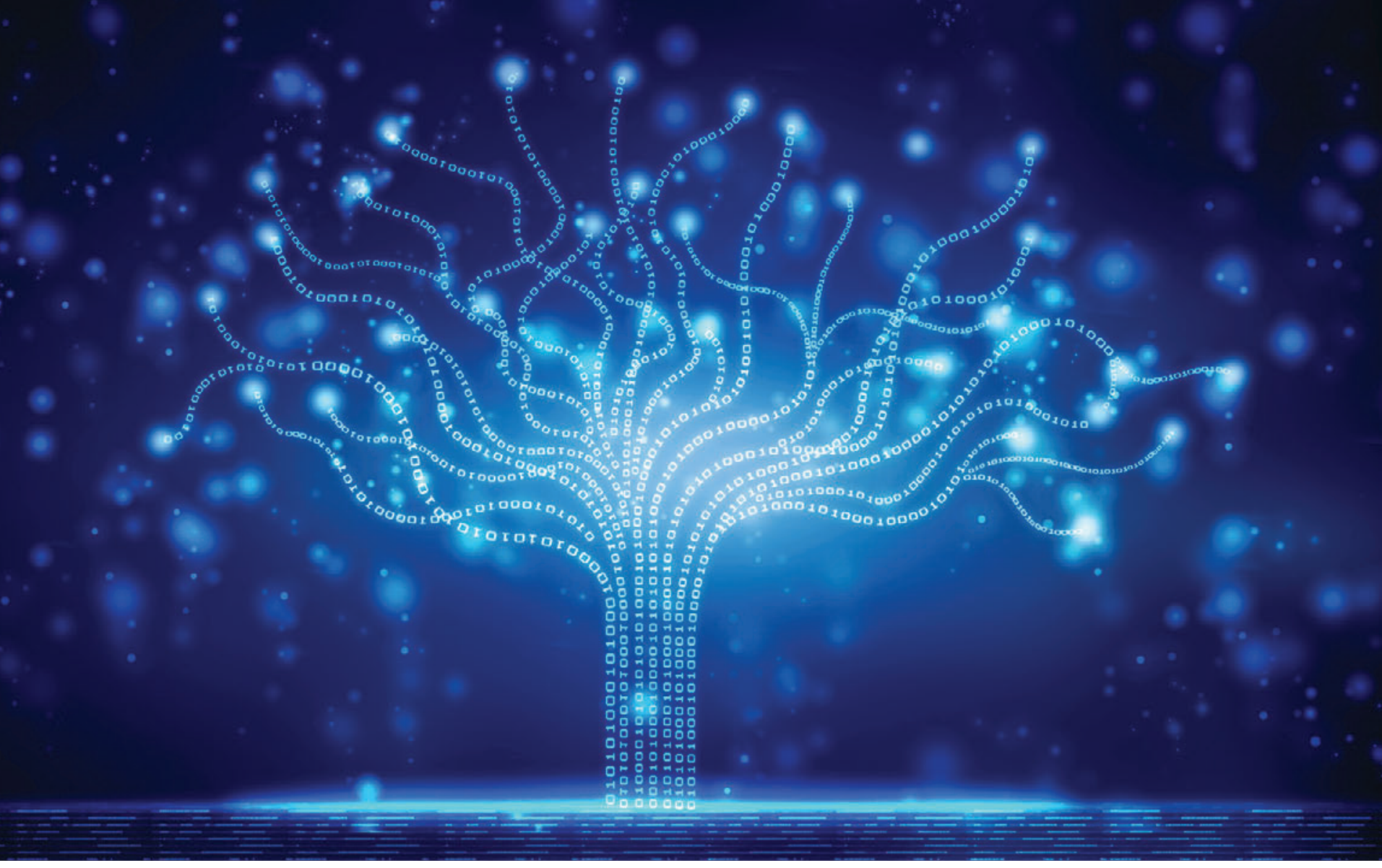

\section{Concluding remarks}

The loT is a rapidly evolving area that has implications for a wide range of industry sectors and stakeholders, especially the public. From our research, it is clear that the development and adoption of the loT presents a number of promising opportunities, particularly in its potential to deliver positive socio-economic benefits, but that there will also be several challenges, particularly around security, privacy and trust. Crucial questions raised in this study will need to be directly addressed through policymaking in the future. These questions relate to how the UK can most effectively enable the deployment of loT products and services to foster business opportunities and shape the loT marketplace, while creating public trust and confidence in the principles by which the loT is governed. The first steps in this direction might involve addressing the questions raised here concerning setting the right framework conditions that ensure long-term growth for the loT, as well as recognising and understanding the nature of the loT as a systemic innovation requiring funding, standards, evidence and trust.

We have closely examined the public policy implications of real loT implementations and user perspectives to provide input to a feedback loop for the whole loT policy community. We hope that using this bottomup approach to engage with and examine the role of two key groups of stakeholders in the loT ecosystem - businesses and individual users of technology - has generated deeper insight for the policy feedback loop. We also propose that the method we deployed in this study can be used in the future to provide a continuous feedback mechanism on how the impact of loT-related policy is progressing in the UK.

This summary describes work done by RAND Europe, commissioned by loTUK and BCS, The Chartered Institute for IT and documented in Accelerating the Internet of Things in the UK: Using Policy to Support Practice by Salil Gunashekar, Anton Spisak, Kevin Dean, Nathan Ryan, Louise Lepetit and Paul Cornish, RR-1492-BCSIOTUK, 2016 (available at www.rand.org/t/rr1492). To view this summary online, visit www.rand.org/t/rb9933. RAND Europe is a not-for-profit research organisation whose mission is to help improve policy and decisionmaking through research and analysis. RAND Europe's publications do not necessarily reflect the opinions of its research clients and sponsors. RAND $^{\circledR}$ is a registered trademark.

Limited Print and Electronic Distribution Rights: This document and trademark(s) contained herein are protected by law. This representation of RAND intellectual property is provided for noncommercial use only. Unauthorised posting of this publication online is prohibited. Permission is given to duplicate this document for personal use only, as long as it is unaltered and complete. Permission is required from RAND to reproduce, or reuse in another form, any of its research documents for commercial use. For information on reprint and linking permissions, please visit www.rand.org/pubs/permissions.html. 